\title{
RESEARCH
}

\section{Free boundary methods and non-scattering phenomena}

\author{
Mikko Salo ${ }^{1^{*}(D)}$ and Henrik Shahgholian ${ }^{2}$
}

\author{
${ }^{*}$ Correspondence: \\ mikko.j.salo@jyu.fi \\ ${ }^{1}$ Department of Mathematics \\ and Statistics, University of \\ Jyväskylä, Jyväskylä, Finland \\ Full list of author information is \\ available at the end of the article
}

\begin{abstract}
We study a question arising in inverse scattering theory: given a penetrable obstacle, does there exist an incident wave that does not scatter? We show that every penetrable obstacle with real-analytic boundary admits such an incident wave. At zero frequency, we use quadrature domains to show that there are also obstacles with inward cusps having this property. In the converse direction, under a nonvanishing condition for the incident wave, we show that there is a dichotomy for boundary points of any penetrable obstacle having this property: either the boundary is regular, or the complement of the obstacle has to be very thin near the point. These facts are proved by invoking results from the theory of free boundary problems.
\end{abstract}

\section{Introduction}

\subsection{Motivation}

In this article, we discuss some examples of non-scattering phenomena based on methods from free boundary problems. The connection between these fields was recently pointed out in [18], and we invoke further ideas from free boundary problems to obtain stronger results. The methods are relevant both for inverse scattering problems and inverse boundary value problems. We first describe the boundary case and state the main results in that setting, and discuss the scattering case afterward. All functions will be assumed real valued unless mentioned otherwise.

Let $\Omega \subset \mathbb{R}^{n}$ be a bounded domain with smooth boundary, and let $q \in L^{\infty}(\Omega)$ be a potential in $\Omega$. Assuming that 0 is not a Dirichlet eigenvalue for $\Delta+q$ in $\Omega$, for any $f \in H^{1 / 2}(\partial \Omega)$, there is a unique solution $u \in H^{1}(\Omega)$ of the Dirichlet problem

$$
(\Delta+q) u=0 \text { in } \Omega,\left.\quad u\right|_{\partial \Omega}=f .
$$

We assume that we can fix a Dirichlet data $f$ and measure the corresponding Neumann data $\left.\partial_{\nu} u\right|_{\partial \Omega}$ (interpreted weakly as an element of $H^{-1 / 2}(\partial \Omega)$ ) on the boundary. This kind of situation arises in diffuse optical tomography [5]. It is also relevant in electrical impedance tomography, i.e., Calderón problem [51], where the underlying conductivity equation $\operatorname{div}(\gamma \nabla v)=0$ can often be reduced to the equation $(\Delta+q) u=0$ with $q=-\gamma^{-1 / 2} \Delta\left(\gamma^{1 / 2}\right)$ by using the Liouville transformation $v=\gamma^{-1 / 2} u$.

(c) The Author(s) 2021, corrected publication 2022. This article is licensed under a Creative Commons Attribution 4.0 International License, which permits use, sharing, adaptation, distribution and reproduction in any medium or format, as long as you give appropriate credit to the original author(s) and the source, provide a link to the Creative Commons licence, and indicate if changes were made. The images or other third party material in this article are included in the article's Creative Commons licence, unless indicated otherwise in a credit line to the material. If material is not included in the article's Creative Commons licence and your intended use is not permitted by statutory regulation or exceeds the permitted use, you will need to obtain permission directly from the copyright holder. To view a copy of this licence, visit http://creativecommons.org/licenses/by/4.0/. 
In inverse boundary value problems of this type, one often assumes the knowledge of the full Dirichlet-to-Neumann map

$$
\Lambda_{q}: H^{1 / 2}(\partial \Omega) \rightarrow H^{-1 / 2}(\partial \Omega), \quad \Lambda_{q} f=\left.\partial_{\nu} u\right|_{\partial \Omega} .
$$

This corresponds to an idealized case where we can perform infinitely many measurements. However, in practice, only finitely many measurements are possible. Moreover, the idealized problem is formally overdetermined when $n \geq 3$, in the sense that the unknown $q$ is a function of $n$ variables, whereas the measurements (Schwartz kernel of $\Lambda_{q}$ ) depend on $2 n-2$ variables. This suggests that fewer measurements might be sufficient. We are interested in the single measurement inverse problem: which properties of $q$ can be determined from the measurement $\left.\partial_{\nu} u\right|_{\partial \Omega}$ corresponding to a fixed Dirichlet data $f \in H^{1 / 2}(\partial \Omega)$ ?

In general, it is not possible to determine a potential $q$ from a single measurement. This is indicated by a heuristic dimension count argument: the measurement $\left.\partial_{\nu} u\right|_{\partial \Omega}$ is a function of $n-1$ variables, whereas the unknown potential is a function of $n$ variables. Thus the inverse problem of determining $q$ from a single measurement is formally underdetermined. A related problem is to determine the shape of a penetrable obstacle from a single measurement. This corresponds to potentials of the form

$$
q=h \chi_{D}, \quad \bar{D} \subset \Omega,
$$

where $\chi_{D}$ is the characteristic function of $D$ (a bounded open set, i.e., the obstacle), and $h$ satisfies a nonvanishing condition at $\partial D$. We will sometimes assume the following conditions for $D$ and $h$.

Definition $A$ bounded open set $D \subset \mathbb{R}^{n}$ is called a solid domain if $D$ and $\mathbb{R}^{n} \backslash \bar{D}$ are connected, and $\operatorname{int}(\bar{D})=D$. We say that $h$ is a contrast for $D$ if

$$
h \in L^{\infty}\left(\mathbb{R}^{n}\right), \quad \text { and }|h| \geq c>0 \text { a.e. in some neighborhood of } \partial D .
$$

Since the potential is $q=h \chi_{D}$, the values of the contrast outside $D$ will not play any role. The inverse problem is to determine the shape of the obstacle, i.e., $\partial D$, or some properties of $\partial D$ from a single measurement $\left.\partial_{\nu} u\right|_{\partial \Omega}$. If $\partial D$ is (say) a Lipschitz domain, then it is locally the graph of a function of $n-1$ variables and thus the inverse problem is formally well-determined.

There are various results in the literature for determining $\partial D$ from a single measurement. For a related Calderón-type problem corresponding to the equation $\operatorname{div}(\gamma \nabla u)=0$ where $\gamma=1+h \chi_{D}$ and $h$ is a nonzero constant, there are partial results when $D$ has special geometry, such as $D$ being a convex polygon or polyhedron, a ball, or a cylinder. There are also local uniqueness results (if $D$ and $D^{\prime}$ are close enough in some sense then they can be distinguished by a single measurement) and estimates for the size of $D$. See [1] for a survey of classical results, and [37] and references therein for more recent results. The results in $[2,6]$ are of particular interest to us: they invoke methods from free boundary problems to show that if $D$ is, e.g., a Lipschitz domain and it is not determined by a single measurement, then part of $\partial D$ is necessarily real-analytic. We refer to $[24,30,31]$ for recent related results.

It turns out that such results are closely connected to a certain non-scattering phenomenon in inverse scattering theory. These problems involve a fixed frequency $\lambda \geq 0$. 
Given a bounded open set $\Omega \subset \mathbb{R}^{n}$, we ask if there exist nontrivial solutions of

$$
\left(\Delta+\lambda^{2}+q\right) u_{q}=0 \quad \text { in } \Omega, \quad\left(\Delta+\lambda^{2}\right) u_{0}=0 \quad \text { in } \Omega
$$

that satisfy

$$
u_{q}, u_{0} \in H^{1}(\Omega), \quad u_{q}-u_{0} \in H_{0}^{2}(\Omega)
$$

This problem is rather similar to the interior transmission problem (see $[16,17])$ but note that we require $u_{q}, u_{0}$ to be $H^{1}$ instead of $L^{2}$. The above problem can in fact be considered as a matching problem as in [25]. One could also accommodate the condition $u_{q}-u_{0}-g \in$ $H_{0}^{2}(\Omega)$ for some smooth enough $g$, which would be close to the inhomogeneous interior transmission problem.

If the boundary of $\Omega$ is smooth enough, the condition (1.2) can be written as

$$
\left.u_{q}\right|_{\partial \Omega}=\left.u_{0}\right|_{\partial \Omega},\left.\quad \partial_{\nu} u_{q}\right|_{\partial \Omega}=\left.\partial_{\nu} u_{0}\right|_{\partial \Omega} .
$$

Thus, if one fixes the Dirichlet data $f=\left.u_{0}\right|_{\partial \Omega}=\left.u_{q}\right|_{\partial \Omega}$, this would mean that the measurement $\left.\partial_{\nu} u_{q}\right|_{\partial \Omega}$ corresponding to $q$ is identical to the measurement $\left.\partial_{\nu} u_{0}\right|_{\partial \Omega}$ for the zero potential. Thus the potential $q$ is invisible for this particular measurement and looks like empty space. In the terminology of scattering theory, if this happens we say that "the incident wave $u_{0}$ does not scatter."

We specialize the above question to the case of an obstacle $D$. The following is the main question studied in this article:

Given a bounded open set $D \subset \mathbb{R}^{n}$ with $\bar{D} \subset \Omega$, is there a contrast $h$ for $D$ such that there exist nontrivial solutions $u_{q}$ and $u_{0}$ with $q=h \chi_{D}$ satisfying (1.1)-(1.2)?

If the answer is positive, then there is some contrast $h$ for $D$ that admits an incident wave that does not scatter (thus $D$ will be invisible with respect to this measurement). On the other hand, if the answer is negative, then the obstacle $D$ scatters every incident wave nontrivially.

\subsection{Main results}

There are various results stating that if $\partial D$ is piecewise smooth and has a corner singularity, then every incident wave will scatter nontrivially. We will give precise references in Sect. 1.4. On the other hand, there seem to be few examples in the literature of penetrable obstacles admitting incident waves that do not scatter. Balls have this property [19, Sections 10.3 and 8.4], and [26] gives examples of potentials $q \in C_{c}^{\infty}\left(\mathbb{R}^{n}\right)$ having this property whose supports are unions of balls. See [53] for some related results.

Our first result states that any obstacle with real-analytic boundary admits incident waves that do not scatter:

Theorem 1.1 Let $\Omega \subset \mathbb{R}^{n}$ be a bounded open set, let $\lambda \geq 0$, and let $D \subset \mathbb{R}^{n}$ be a bounded open set with real-analytic boundary such that $\bar{D} \subset \Omega$ and $\mathbb{R}^{n} \backslash \bar{D}$ is connected. Suppose that $\lambda$ is not a Dirichlet eigenvalue for $-\Delta$ in $D$. Then, there is a contrast for $D$ that admits an incident wave that does not scatter.

While corner singularities typically scatter every incident wave, we show that at least for $\lambda=0$ there also exist obstacles with inward cusp singularities admitting incident 
waves that do not scatter. We say that a connected open set $D \subset \mathbb{R}^{n}$ is a quadrature domain (for harmonic functions) if there is a compactly supported distribution $\mu$ in $\mathbb{R}^{n}$ with $\operatorname{supp}(\mu) \subset D$ such that

$$
\int_{D} H d x=\int_{D} H d \mu
$$

whenever $H \in L^{1}(D)$ is harmonic in $D$. A basic example is a ball $B(a, r) \subset \mathbb{R}^{n}$ with $\mu=\left|B_{r}\right| \delta_{a}$, so that (1.3) holds by the mean value theorem. There exist many examples of quadrature domains, and their boundaries can exhibit inward cusps (see [21, Chapter 14] or [46] for examples). One example is the cardioid domain $D=\left\{w+\frac{1}{2} w^{2}: w \in \mathbb{D}\right\} \subset \mathbb{R}^{2}$ that has an inward cusp, see [44, Figure 0.1] (though note that $\partial D$ is the image of $S^{1}$ by an analytic map).

Theorem 1.2 Let $\Omega \subset \mathbb{R}^{n}$ be a bounded open set, let $\lambda=0$, and let $D \subset \mathbb{R}^{n}$ be a quadrature domain such that $\bar{D} \subset \Omega$. Then there is a contrast for $D$ that admits an incident wave that does not scatter.

As non-scattering incident waves in Theorems 1.1 and 1.2, one can choose any solution of

$$
\left(\Delta+\lambda^{2}\right) u_{0}=0 \quad \text { in } \Omega
$$

such that $u_{0}$ is positive on $\partial D$. A nonvanishing condition for $u_{0}$ on $\partial D$ will be important for many results in this article (with the exception of Theorem 1.6), and it is of interest to determine if such solutions $u_{0}$ exist. They always do when $\lambda=0$ (take $u_{0} \equiv 1$ ) or when $u_{0}$ is allowed to be complex valued (take $u_{0}=e^{i \lambda x_{1}}$ ). However, by Lemma 3.1 any real-valued solution of (1.4) has a zero in any ball of radius $\geq c_{n} / \lambda$ and the nonvanishing condition on $\partial D$ is nontrivial in this case. In fact, if $\lambda$ is a Dirichlet eigenvalue of $-\Delta$ in $D$ then solutions $u_{0}$ satisfying the nonvanishing condition may not exist (see Remark 3.2). On the positive side, we will show the following result.

Theorem 1.3 Let $D$ be a bounded $C^{1}$ domain, or Lipschitz domain when $n=2,3$, with $\mathbb{R}^{n} \backslash \bar{D}$ connected. Suppose that $\lambda>0$ is not a Dirichlet eigenvalue of $-\Delta$ in $D$. Then, there is a real-valued solution $u_{0}$ of $\left(\Delta+\lambda^{2}\right) u_{0}=0$ in $\mathbb{R}^{n}$ such that $u_{0}$ is positive on $\partial D$.

Theorems 1.1 and 1.2 are not difficult to prove, and they are analogous to certain facts in the theory of free boundary problems. As mentioned above, the connection between single measurement inverse problems and free boundary methods is classical in the Calderón problem $[2,6]$. Curiously, it seems that for non-scattering phenomena, this connection was only pointed out very recently in [18]. The main point is the following: if an obstacle $D$ admits an incident wave that does not scatter, then $\partial D$ can be understood as a free boundary in a certain obstacle-type problem. This observation was used in [18] to show that if $D$ has Lipschitz boundary and the incident wave $u_{0}$ is nonvanishing on $\partial D$, then necessarily $\partial D$ must be real-analytic (resp. $C^{k+1, \alpha}$ ) if the contrast is real-analytic (resp. $\left.C^{k, \alpha}\right)$.

We prove a corresponding result where the a priori assumption that $D$ has Lipschitz boundary is removed. However, as indicated by Theorem 1.2, one must then allow for the 
possibility that $D$ has inward cusps. We first need to introduce the concept of minimal diameter.

For any set $K$, we define $\operatorname{MD}(K)$ to be the minimal diameter of $K$, i.e., the infimum of distances between pairs of parallel planes such that $K$ is contained in the strip determined by the planes. For any ball $B(z, r)$, we also define the thickness function

$$
\delta_{r}(K, z):=\frac{\operatorname{MD}(K \cap B(z, r))}{r} .
$$

To illustrate this notion, note that if $D \subset \mathbb{R}^{n}$ is a bounded Lipschitz domain, then there are $c, r_{0}>0$ such that

$$
\delta_{r}\left(\mathbb{R}^{n} \backslash D, x^{0}\right) \geq c \quad \text { whenever } x^{0} \in \partial D \text { and } 0<r<r_{0} .
$$

On the other hand, if $D \cap B(0,1)=\left\{x_{n}<\left|x^{\prime}\right|^{1 / \gamma}\right\} \cap B(0,1)$ where $\gamma>1$, so that $D$ has an inward cusp at 0 , one can check that

$$
\delta_{r}\left(\mathbb{R}^{n} \backslash D, 0\right) \leq C r^{\gamma-1}, \quad 0<r<1 .
$$

We first state the following result showing that if $D$ admits an incident wave $u_{0}$ that does not scatter, and if both the contrast $h$ and $u_{0}$ are nonvanishing at a point $x^{0} \in \partial D$, then there are two possibilities: either $D$ is regular near $x^{0}$, or the complement of $D$ is thin near $x^{0}$.

Theorem 1.4 Let $\Omega \subset \mathbb{R}^{n}$ be a bounded open set, and suppose that $u_{q}, u_{0} \in H^{1}(\Omega)$ satisfy (1.1)-(1.2) where $q=h \chi_{D}$ for some solid domain $D$ with $\bar{D} \subset \Omega$. Assume that $h$ is Dini continuous (i.e., $h$ has a modulus of continuity $\omega$ with $\left.\int_{0}^{1} \omega(r) d(\log r)<\infty\right)$. For any $x^{0} \in \partial D$ such that

$$
h\left(x^{0}\right) u_{0}\left(x^{0}\right) \neq 0,
$$

one of the following conditions holds:

(a) $\lim \sup _{r \rightarrow 0} \delta_{r}\left(\Omega \backslash D, x^{0}\right)>0$, and $D$ is locally a $C^{1}$ domain near $x^{0}$; or

(b) $\Omega \backslash D$ is thin near $x^{0}$ in the sense that $\lim _{r \rightarrow 0} \delta_{r}\left(\Omega \backslash D, x^{0}\right)=0$.

If $h$ is additionally assumed to be Lipschitz (resp. $C^{k, \alpha}$ where $k \geq 1$ and $0<\alpha<1$, or realanalytic) near $x^{0}$ and if (a) holds, then $D$ is locally a $C^{1, \alpha}$ (resp. $C^{k+1, \alpha}$, or real-analytic) domain near $x^{0}$.

The case where $h$ is Dini continuous is a consequence of the following more precise result from [4, Theorem 1.3]:

Theorem 1.5 Retain the hypotheses of Theorem 1.4, with h being a Dini continuous function. Suppose for some $r_{1}>0, x^{0} \in \partial D$, we have

$$
h(x) u_{0}(x) \neq 0 \quad \text { for } \quad x \in B\left(x^{0}, r_{1}\right) .
$$

Then, there exists a modulus of continuity $\sigma(r)$, and a universal constant $\tau>0$, such that if for some $0<r_{0}<r_{1}$, we have $\sigma\left(r_{0}\right)<\delta_{r_{0}}\left(\left\{u_{0}=u_{q}\right\}, x^{0}\right)$ then $\partial D \cap B\left(x^{0}, \tau r_{0}\right)$ is a $C^{1}$-graph.

If $h$ is Lipschitz, or if $h$ is $C^{1,1}$ and $u_{0}$ vanishes on $\partial D$ but $\nabla u_{0}$ is nonvanishing, we also have the following result. 
Theorem 1.6 Retain the hypotheses of Theorem 1.4, and suppose that either of the following conditions is satisfied:

1. $h \in C^{0,1}\left(B\left(x^{0}, r\right)\right)$,

2. $h \in C^{1,1}\left(B\left(x^{0}, r\right)\right)$ and condition (1.5) is replaced by

$$
|h|>0 \text { and } u_{0}=0 \text { and }\left|\nabla u_{0}\right|>0 \text { on } \partial D \cap B\left(x^{0}, r\right)
$$

Then, there exists $r_{0}>0$ such that one of the following holds:

(a) $\partial D$ is a $C^{1, \alpha}$ graph in $B\left(x^{0}, r_{0}\right)$.

(b) In a translated and rotated system of coordinates

$$
\partial D \cap B\left(x^{0}, r_{0}\right) \subset\left\{x:\left|x_{1}\right|<k\left(x^{\prime}\right)\right\}
$$

where $x^{\prime}=\left(x_{2}, \cdots, x_{n}\right)$, and $k\left(x^{\prime}\right) \geq 0$ is a $C^{1}$-function with $k\left(0^{\prime}\right)=0$.

It is noteworthy that Theorem 1.6 can be "calibrated" to the case where $u_{0}$ vanishes to a fixed higher order on some part of $\partial D$, by asking higher-order regularity for the righthand side. Notwithstanding this, it remains a tantalizing problem when the higher-order vanishing of $u_{0}$ takes place on isolated points of $\partial D$. This remains to be studied in the future. See [56] for partial results in this direction.

\subsection{Connection to free boundary problems}

We now describe more precisely how the existence of an incident wave that does not scatter leads to a free boundary problem. Let $\Omega \subset \mathbb{R}^{n}$ be a bounded open set, and suppose that $u_{q}, u_{0} \in H^{1}(\Omega)$ satisfy (1.1)-(1.2) where $q=h \chi_{D}$ for some solid domain $D$ with $\bar{D} \subset \Omega$ and for $h \in C\left(\mathbb{R}^{n}\right)$. Then, $u_{0}$ is real-analytic, and also $u_{q} \in W_{\text {loc }}^{2, p}(\Omega)$ for any $p<\infty$ by elliptic regularity. We write $u:=u_{q}-u_{0} \in H_{0}^{2}(\Omega)$ and extend $u$ by zero to $\mathbb{R}^{n}$. Then, $u \in H^{2}\left(\mathbb{R}^{n}\right)$ satisfies

$$
\left(\Delta+\lambda^{2}\right) u=f_{0} \chi_{D} \quad \text { in } \mathbb{R}^{n}
$$

where $f_{0}=-h u_{q}$ near $\bar{D}$. Note also that since $u$ solves $\left(\Delta+\lambda^{2}\right) u=0$ in $\mathbb{R}^{n} \backslash \bar{D}$ and $\left.u\right|_{\mathbb{R}^{n} \backslash \bar{\Omega}}=0$, unique continuation implies that $\left.u\right|_{\mathbb{R}^{n} \backslash \bar{D}}=0$ using that $\mathbb{R}^{n} \backslash \bar{D}$ is connected. Suppose that

$$
h(z) u_{0}(z) \neq 0 \quad \text { at some } z \in \partial D .
$$

Since $u_{q}=u_{0}$ outside $\bar{D}$ and $u_{q}$ is continuous, we also have $f_{0}(z) \neq 0$. We claim that

$$
\bar{D} \cap B(z, r)=\operatorname{supp}(u) \cap B(z, r)
$$

whenever $r>0$ is such that $\left|h u_{q}\right|>0$ in $B(z, r)$. Since $\left.u\right|_{\mathbb{R}^{n} \backslash \bar{D}}=0$, one always has $\operatorname{supp}(u) \cap B(z, r) \subset \bar{D} \cap B(z, r)$. Conversely, let $x \in \bar{D} \cap B(z, r)$. If $x \notin \operatorname{supp}(u)$, then $u=0$ near $x$, which by (1.7) implies that $f_{0}=0$ near $x$ which is impossible since $\left|f_{0}\right|>0$ in $B(z, r)$. This proves (1.9).

Since $D$ is a solid domain, it follows from (1.9) that

$$
D \cap B(z, r)=\operatorname{int}(\operatorname{supp}(u)) \cap B(z, r) .
$$


Thus, (1.7) implies that

$$
\left(\Delta+\lambda^{2}\right) u=f_{0} \chi_{\operatorname{int}(\operatorname{supp}(u))} \text { in } B(z, r), \quad z \in \partial(\operatorname{int}(\operatorname{supp}(u)))
$$

Writing $f=f_{0}-\lambda^{2} u$, so that $f(z)=f_{0}(z) \neq 0$, we may further write this as

$$
\Delta u=f \chi_{\operatorname{int}(\operatorname{supp}(u))} \text { in } B(z, r), \quad z \in \partial(\operatorname{int}(\operatorname{supp}(u))) .
$$

The last equation only involves $u$ and not $D$. Thus, we have reduced our original problem to an obstacle problem in free boundary theory, where locally near $z$ the obstacle is the set $\operatorname{int}(\operatorname{supp}(u))$ and its boundary $\partial(\operatorname{int}(\operatorname{supp}(u)))$ can be understood as a free boundary. In the free boundary literature, it is more customary to work with equations like

$$
\Delta u=f \chi_{\{u \neq 0\}} \text { in } B(z, r), \quad z \in \partial(\{u=|\nabla u|=0\}) .
$$

However, the methods for proving regularity of the free boundary in (1.12) also apply to (1.11). See [44] for even more general equations when $f$ is assumed Lipschitz, or [4] for $f$ Dini.

The standard obstacle problem corresponds to (1.12) for solutions $u \geq 0$ (and $f \equiv 1$ in the most classical case). In our case, $u=u_{q}-u_{0}$, and it is not possible to assume that $u$ is nonnegative. This means that (1.12) corresponds to a no-sign obstacle problem. The no-sign assumption on $u$ makes the analysis of this problem extremely hard, and one has to resort to advanced tools such as monotonicity formulas along with strong geometric analysis. On the other hand, if $\partial D$ is Lipschitz close to $z$, then it follows by standard free boundary techniques that $u$ has a sign in a vicinity of $z$; see the beginning of the proof of Theorem 1.3 in [4]. Thus, the case of Lipschitz domains falls back to the regularity theory for the standard obstacle problem, which is rather classical [13].

There is by now also a well-developed theory for no-sign obstacle problems when $f$ is nonvanishing at the point $z$ of interest, i.e., when (1.8) holds. We refer to [44] for an account of this theory. After the reduction to (1.11), Theorems 1.4-1.6 follow rather directly from this theory. One can also accommodate the possibility that $f$ vanishes to some fixed order at each point of $\partial D \cap B(z, r)$ (see Theorem 1.6 for an example result). If $f$, or $u_{0}$, only vanishes at $z$ (or in a set of dimension $\leq n-2$ ), then the problem becomes non-standard and is more or less untouched in the free boundary literature.

\subsection{Inverse scattering}

Finally, we discuss the case of inverse scattering problems where the bounded domain $\Omega$ is replaced by $\mathbb{R}^{n}$. In this subsection, we allow functions to be complex valued.

Let $\lambda>0$ be a fixed frequency, and let $u_{0}$ be a solution of $\left(\Delta+\lambda^{2}\right) u_{0}=0$ in $\mathbb{R}^{n}$. We consider $u_{0}$ as an incident wave that is used to probe a medium whose scattering properties are described by a compactly supported potential $q \in L^{\infty}\left(\mathbb{R}^{n}\right)$. The incident wave $u_{0}$ induces a total wave $u_{q}=u_{0}+v$ that solves

$$
\left(\Delta+\lambda^{2}+q\right) u_{q}=0 \quad \text { in } \mathbb{R}^{n} .
$$

The solution is unique if we require that the scattered wave $v$ is outgoing in the sense that

$$
v=\left(-\Delta-(\lambda+i 0)^{2}-q\right)^{-1}\left(q u_{0}\right)
$$


where $\left(-\Delta-(\lambda+i 0)^{2}-q\right)^{-1}$ is the outgoing resolvent. Since $q u_{0}$ is compactly supported, $v$ has the asymptotics

$$
v(r \theta)=e^{i \lambda r} r^{-\frac{n-1}{2}} u_{q}^{\infty}(\theta)+o\left(r^{-\frac{n-1}{2}}\right) \quad \text { as } r \rightarrow \infty,
$$

where $\theta \in S^{n-1}$. The function $u_{q}^{\infty}$ on $S^{n-1}$ is called the far field pattern corresponding to incident wave $u_{0}$, and it can be measured from the knowledge of $u_{q}$ as $|x| \rightarrow \infty$. We refer to $[19,55]$ for these basic facts.

A commonly used class of incident waves is given by the Herglotz waves, which are solutions of $\left(\Delta+\lambda^{2}\right) u_{0}=0$ having the form

$$
u_{0}(x)=\int_{S^{n-1}} e^{i \lambda x \cdot \omega} f(\omega) d \omega, \quad f \in L^{2}\left(S^{n-1}\right)
$$

A scattering analogue of the Dirichlet-to-Neumann map is given by the far field operator

$$
A_{q}(\lambda): L^{2}\left(S^{n-1}\right) \rightarrow L^{2}\left(S^{n-1}\right), \quad A_{q}(\lambda) f=u_{q}^{\infty} .
$$

A standard fixed frequency inverse problem is to determine $q$ from the knowledge of $A_{q}(\lambda)$, which corresponds to infinitely many measurements. However, we wish consider the single measurement problem in (fixed frequency) inverse scattering: determine some properties of $q$ from knowledge of the far field pattern $u_{q}^{\infty}$ corresponding to a fixed incident wave $u_{0}$. If $u_{q}^{\infty} \equiv 0$, we say that the incident wave $u_{0}$ does not scatter. Again, in order to obtain a formally well-determined problem, we consider the case of penetrable obstacles, so that

$$
q=h \chi_{D}
$$

where $D \subset \mathbb{R}^{n}$ is a bounded open set (the obstacle) and $h$ is a contrast for $D$.

In the imaging community, it has been understood for a long time that if $\partial D$ has corner singularities, one often has strong scattering effects. A rigorous analysis of this phenomenon was initiated in the important work [11] which showed that if part of $\partial D$ is part of a cube, then every incident wave scatters nontrivially for every frequency $\lambda>0$. In two dimensions, this was extended to sectors with angle $<90^{\circ}$ and single measurement results in $[27,43]$. The analysis was based on studying Laplace transforms of characteristic functions of cones via complex geometrical optics solutions as in the Calderón problem. There are several related results including quantitative bounds even when corners are replaced by high curvature points, see [7-10,12] and the survey [36] (which also discusses results for electromagnetic and elastic scattering). Another important approach to nonscattering problems, introduced in [23] (see [22,35] for related work), is based on the theory of boundary value problems in corner domains and can be used to produce similar results even for curvilinear polyhedra or when $h$ vanishes to finite order at $\partial D$. We mention that these results related to corner singularities are most complete for $n=2$, and even when $n=3$ they become more limited and mostly apply to edge or circular cone singularities. Finally, the results in [18], already discussed before, show regularity of the free boundary if the obstacle is a Lipschitz domain, and the incident wave is nonvanishing on its boundary.

In [11], a frequency $\lambda>0$ was called a non-scattering wavenumber if there is some incident wave $u_{0}$ that does not scatter. The results mentioned above show that if $\partial D$ has corner singularities, then there are no non-scattering wavenumbers (i.e., every incident wave 
scatters nontrivially independent of the frequency). This notion is connected with interior transmission eigenvalues (see $[16,17]$ ) in the sense that a non-scattering wavenumber is also an interior transmission eigenvalue. The converse is not true: for a given potential, there are typically infinitely many interior transmission eigenvalues whereas the set of non-scattering frequencies may be empty.

The free boundary approach described above extends directly to the scattering case. If $u_{0}$ is an incident wave solving $\left(\Delta+\lambda^{2}\right) u_{0}=0$ in $\mathbb{R}^{n}$, let $u_{q}$ be the outgoing solution of $\left(\Delta+\lambda^{2}+q\right) u_{q}=0$ in $\mathbb{R}^{n}$ defined above where $q=h \chi_{D}$, and $D$ is a bounded solid domain. Suppose that $u_{0}$ does not scatter, i.e., $u_{q}^{\infty} \equiv 0$. Then, the Rellich uniqueness theorem and unique continuation imply that $u_{q}=u_{0}$ in $\mathbb{R}^{n} \backslash \bar{D}$. We may thus take $\Omega$ to be some large ball containing $\bar{D}$, and we are back in the situation of (1.1)-(1.2). Moreover, if $h$ is assumed to be real valued, and if for some $z \in \partial D$, one has $h(z) u_{0}(z) \neq 0$, then $\operatorname{Re}\left(u_{0}\right)(z) \neq 0$ or $\operatorname{Im}\left(u_{0}\right)(z) \neq 0$. Thus by taking real or imaginary parts of the solutions, one can reduce to a situation where the functions involved are real valued.

If the obstacle has real-analytic boundary, combining Theorem 2.1 (with $\Omega=\mathbb{R}^{n}$ ) and Theorem 1.3 leads to an analogue of Theorem 1.1 in the scattering setting. This provides examples of real-valued contrasts and incident Herglotz waves that do not scatter.

\section{Structure of the article}

In Sect. 2, we will prove Theorems 1.1 and 1.2 by using a simple extension argument, the Cauchy-Kowalevski theorem and the defining property of quadrature domains. In Sect. 3, we discuss Helmholtz solutions and prove Theorem 1.3, which follows by combining a result in $D$ with a Runge approximation argument. In Sect. 4, we discuss how Theorems 1.4-1.6 follow from arguments in the theory of free boundaries.

\section{Examples of free boundaries}

In this section, we show that any real-analytic boundary, or the boundary of any quadrature domain when $\lambda=0$, can be realized as a free boundary. The following results are more precise versions of Theorems 1.1 and 1.2, since they also give information on the kinds of incident waves and contrasts for which one has no scattering. Note that Theorem 1.1 follows by combining Theorems 2.1 and 1.3 , and 1.2 follows from Theorem 2.2 by taking $u_{0} \equiv 1$.

Theorem 2.1 Let $\lambda \geq 0$, let $D \subset \mathbb{R}^{n}$ be a bounded open set with real-analytic boundary, let $\Omega \subset \mathbb{R}^{n}$ be an open set with $\bar{D} \subset \Omega$, and let $u_{0}$ be any solution of $\left(\Delta+\lambda^{2}\right) u_{0}=0$ in $\Omega$ that is positive on $\partial D$. Let also $h_{0}$ be a real-analytic function near $\partial D$. Then, there is $h \in C^{\infty}(\bar{\Omega})$ with $h=h_{0}$ near $\partial D$ so that with the choice $q=h \chi_{D}$, the equation

$$
\left(\Delta+\lambda^{2}+q\right) u_{q}=0 \quad \text { in } \Omega
$$

has a solution $u_{q} \in H^{2}(\Omega)$ satisfying $u_{q}=u_{0}$ in $\Omega \backslash \bar{D}$.

Theorem 2.2 Let $D \subset \mathbb{R}^{n}$ be a bounded quadrature domain, let $\Omega \subset \mathbb{R}^{n}$ be an open set with $\bar{D} \subset \Omega$, and let $u_{0}$ be any solution of $\Delta u_{0}=0$ in $\Omega$ that is positive on $\partial D$. Then, there is $h \in L^{\infty}(\Omega)$ with $|h| \geq c>0$ near $\partial D$ so that with the choice $q=h \chi_{D}$, the equation

$$
(\Delta+q) u_{q}=0 \quad \text { in } \Omega
$$


has a solution $u_{q} \in H^{2}(\Omega)$ satisfying $u_{q}=u_{0}$ in $\Omega \backslash \bar{D}$.

We mention that the existence of solutions of $\left(\Delta+\lambda^{2}\right) u_{0}=0$ in $\mathbb{R}^{n}$ that are positive on $\partial D$ is proved later in Proposition 3.3. It follows from the proof that $u_{0}$ can be chosen to be a Herglotz wave, i.e., of the form (1.13), which is relevant for applications in scattering theory.

The proofs of Theorems 2.1 and 2.2 involve the following simple result. It begins with a solution of $\left(\Delta+\lambda^{2}\right) u_{0}=0$ in $\Omega$ that is positive on $\partial D$ and with a local solution $v_{0}$, for some potential $h_{0}$ that extends $u_{0}$ slightly inside $D$. The result gives a solution $v$ that extends $u_{0}$ all the way into $D$ and corresponds to some potential $h \chi_{D}$, where $h$ extends $h_{0}$ into $D$. The point is that one first chooses a suitable extension $v$ of $v_{0}$, and then constructs the potential $h$ depending on $v$.

Lemma 2.3 Let $\Omega \subset \mathbb{R}^{n}$ be open, let $D \subset \mathbb{R}^{n}$ be a bounded open set with $\bar{D} \subset \Omega$, let $\lambda \geq 0$, and assume that $\left(\Delta+\lambda^{2}\right) u_{0}=0$ in $\Omega$ with $u_{0}$ positive on $\partial D$. Suppose that $U$ is a neighborhood of $\partial D$ in $\Omega$ and that $h_{0} \in L^{\infty}(U)$ and $v_{0} \in C^{1,1}(U)$ satisfy

$$
\left(\Delta+\lambda^{2}+h_{0}\right) v_{0}=0 \text { in } D \cap U,\left.\quad v_{0}\right|_{U \backslash \bar{D}}=\left.u_{0}\right|_{U \backslash \bar{D}} .
$$

Then, there are $h \in L^{\infty}(\Omega)$ and $v \in C^{1,1}(\Omega)$, with $h=h_{0}$ and $v=v_{0}$ near $\partial D$, so that

$$
\left(\Delta+\lambda^{2}+h \chi_{D}\right) v=0 \text { in } \Omega,\left.\quad v\right|_{\Omega \backslash \bar{D}}=\left.u_{0}\right|_{\Omega \backslash \bar{D}} .
$$

If additionally $h_{0} \in C^{\infty}(U)$ and $\left.v_{0}\right|_{\bar{D} \cap U} \in C^{\infty}(\bar{D} \cap U)$, then $h \in C^{\infty}(\bar{\Omega})$.

Proof Note that $v_{0}$ is positive in some neighborhood $U_{1} \subset U$ of $\partial D$, since $v_{0} \in C^{1,1}(U)$ and $\left.v_{0}\right|_{U \backslash \bar{D}}=\left.u_{0}\right|_{U \backslash \bar{D}}$ and $u_{0}$ is positive on $\partial D$. Let $\psi \in C_{c}^{\infty}\left(U_{1}\right)$ satisfy $0 \leq \psi \leq 1$ and $\psi=1$ near $\partial D$, and define

$$
v= \begin{cases}v_{0} \psi+(1-\psi) & \text { in } \bar{D}, \\ u_{0} & \text { in } \Omega \backslash \bar{D} .\end{cases}
$$

Then, $v \in C^{1,1}(\Omega)$ is positive near $\bar{D}$ and satisfies $v=v_{0}$ near $\partial D$. One can now define a function $h \in L^{\infty}(\Omega)$ by

$$
h=\left\{\begin{aligned}
-\frac{\left(\Delta+\lambda^{2}\right) v}{v} & \text { in } \bar{D}, \\
\psi h_{0} & \text { in } \Omega \backslash \bar{D} .
\end{aligned}\right.
$$

The functions $h$ and $v$ will have the required properties.

If additionally $h_{0} \in C^{\infty}(U)$ and $\left.v_{0}\right|_{\bar{D} \cap U} \in C^{\infty}(\bar{D} \cap U)$, it follows that $\left.v\right|_{\bar{D}} \in C^{\infty}(\bar{D})$ and $\left.h\right|_{\bar{D}} \in C^{\infty}(\bar{D})$. Since $h=h_{0}$ near $\partial D$, we have $h \in C^{\infty}(\bar{\Omega})$.

By Lemma 2.3, the proofs of Theorems 2.1 and 2.2 are reduced to finding a local solution $v_{0}$ that extends $u_{0}$ a little bit inside $D$. In the real-analytic case, this can be done by solving a Cauchy problem using the Cauchy-Kowalevski theorem.

Proof of Theorem 2.1 Note that $u_{0}$ is real-analytic in $\Omega$. For any $x \in \partial D$, we may use the Cauchy-Kowalevski theorem to find a real-analytic solution of

$$
\left(\Delta+\lambda^{2}+h_{0}\right) v_{x}=0 \text { in } U_{x},\left.\quad v_{x}\right|_{U_{x} \cap \partial D}=\left.u_{0}\right|_{U_{x} \cap \partial D},\left.\quad \partial_{\nu} v_{x}\right|_{U_{x} \cap \partial D}=\left.\partial_{\nu} u_{0}\right|_{U_{x} \cap \partial D},
$$

where $U_{x}$ is an open set of the form $\left\{z+t v(z): z \in V_{x},|t|<\varepsilon_{x}\right\}$ with $V_{x}$ a neighborhood of $x$ in $\partial D$ and $\varepsilon_{x}>0$. Any two solutions $v_{x}$ and $v_{y}$ agree on their overlap $U_{x} \cap U_{y}$ by 
the unique continuation principle. Thus, for some neighborhood $U$ of $\partial D$ in $\Omega$, there is a real-analytic function $v_{0}$ in $U$ so that

$$
\left(\Delta+\lambda^{2}+h_{0}\right) v_{0}=0 \text { in } U,\left.\quad v_{0}\right|_{\partial D}=\left.u_{0}\right|_{\partial D},\left.\quad \partial_{\nu} v_{0}\right|_{\partial D}=\left.\partial_{\nu} u_{0}\right|_{\partial D} .
$$

We may redefine $v_{0}=u_{0}$ in $U \backslash \bar{D}$, so that $v_{0} \in C^{1,1}(U)$ will satisfy

$$
\left(\Delta+\lambda^{2}+h_{0}\right) v_{0}=0 \text { in } D \cap U,\left.\quad v_{0}\right|_{U \backslash \bar{D}}=u_{0} .
$$

By Lemma 2.3, there are $v \in C^{1,1}(\Omega)$ and $h \in C^{\infty}(\bar{\Omega})$ that satisfy $v=v_{0}$ and $h=h_{0}$ near $\partial D$, such that

$$
\left(\Delta+\lambda^{2}+h \chi_{D}\right) v=0 \text { in } \Omega,\left.\quad v_{0}\right|_{\Omega \backslash \bar{D}}=u_{0} .
$$

It remains to set $u_{q}=v$ in $D$ and $u_{q}=u_{0}$ in $\Omega \backslash \bar{D}$. Then, $u_{q} \in H^{2}(\Omega)$ has the required properties.

In the case of quadrature domains, we instead use (1.3) to produce the required local solution.

Proof of Theorem 2.2 Let $G$ be the fundamental solution for $-\Delta$ in $\mathbb{R}^{n}$, i.e. $G(x)=c_{2} \log |x|$ when $n=2$ and $G(x)=c_{n}|x|^{2-n}$ when $n \geq 3$. Let $\mu$ be the distribution with $\operatorname{supp}(\mu) \subset D$ appearing in the definition of the quadrature domain $D$, and define

$$
u=G *\left(\chi_{D}-\mu\right) .
$$

Since $\chi_{D}-\mu$ is a compactly supported distribution, $u$ is a distribution in $\mathbb{R}^{n}$, and it satisfies

$$
\Delta u=\chi_{D}-\mu \text { in } \mathbb{R}^{n} .
$$

Moreover, if $x \in \mathbb{R}^{n} \backslash \bar{D}$, we may take $H(y)=G(x-y)$ in (1.3) to obtain that

$$
\left.u\right|_{\mathbb{R}^{n} \backslash \bar{D}}=0 .
$$

In particular, since $\operatorname{supp}(\mu) \subset D$, there is a neighborhood $U$ of $\partial D$ in $\mathbb{R}^{n}$ such that

$$
\Delta u=\chi_{D} \text { in } U,\left.\quad u\right|_{U \backslash \bar{D}}=0 .
$$

Note that $u \in C^{1,1}(U)$ using the $C^{1,1}$ regularity results for the no-sign obstacle problem [4].

Define $v_{0}=u+u_{0}$ in $U$. We first claim that there is $h_{0}$ near $\partial D$ with $\left|h_{0}\right| \geq c>0$ near $\partial D$ so that

$$
\left(\Delta+h_{0} \chi_{D}\right) v_{0}=0 \text { near } \partial D .
$$

In fact, using the equations for $u$ and $u_{0}$, for any $h_{0}$ one has

$$
\left(\Delta+h_{0} \chi_{D}\right) v_{0}=\chi_{D}+h_{0} \chi_{D} v_{0} \text { in } U .
$$

This quantity vanishes near $\partial D$, if we set

$$
h_{0}=-\frac{1}{v_{0}} \text { near } \partial D \text {. }
$$

The denominator is nonvanishing near $\partial D$ since $u$ is continuous with $\left.u\right|_{\partial D}=0$, and since $u_{0}$ is positive on $\partial D$. We have thus found the required function $h_{0}$ near $\partial D$.

By Lemma 2.3, there are $h \in L^{\infty}(\Omega)$ and $v \in C_{\operatorname{loc}}^{1,1}(\Omega)$, with $h=h_{0}$ and $v=v_{0}$ near $\partial D$, so that

$$
\left(\Delta+h \chi_{D}\right) v=0 \text { in } \Omega,\left.\quad v\right|_{\Omega \backslash \bar{D}}=\left.u_{0}\right|_{\Omega \backslash \bar{D}} .
$$

Setting $u_{q}=v$ in $\bar{D}$ and $u_{q}=u_{0}$ in $\Omega \backslash \bar{D}$ gives the required solution. 
Remark 2.4 The only property of quadrature domains needed in the proof of Theorem 2.2 was the existence of a function $u$ satisfying (2.1). The conclusion of Theorem 2.2, also with a frequency $\lambda \geq 0$, would hold for any domain $D$ that admits a function $u$ satisfying

$$
\left(\Delta+\lambda^{2}\right) u=f \chi_{D} \text { in } U,\left.\quad u\right|_{U \backslash \bar{D}}=0,
$$

where $f$ is nonvanishing on $\partial D$. In Theorem 2.2, we produced such a function as $u=$ $G *\left(\chi_{D}-\mu\right)$, which can be understood as continuing the potential $G * \chi_{D}$ smoothly a little bit inside $D$ as the function $G * \mu$.

\section{Zero sets of Helmholtz solutions}

We complement the previous results by showing that for any bounded open set $D$, there is a solution $u_{0}$ of $\left(\Delta+\lambda^{2}\right) u_{0}=0$ in $\mathbb{R}^{n}$ which is positive on $\partial D$, under some restrictions on $D$ and $\lambda$. In the case $\lambda=0$, one can take $u_{0} \equiv 1$, so we will assume $\lambda>0$. If one allows complex-valued solutions, the function $u_{0}=e^{i \lambda x_{1}}$ is a nonvanishing solution in $\mathbb{R}^{n}$. However, real-valued solutions always have zeros. This is already seen in the case $n=1$ where any solution of $\left(\Delta+\lambda^{2}\right) u_{0}=0$ takes the form $u_{0}(x)=a \sin (\lambda x)+b \cos (\lambda x)$, and such a function has a zero in any closed interval of length $\pi / \lambda$. A similar result holds for Laplace eigenfunctions in compact manifolds (see the survey [38]). The following version of this result shows that any real solution of $\left(\Delta+\lambda^{2}\right) u_{0}=0$ in $\mathbb{R}^{n}$ has a zero in any ball of radius $\geq c_{n} / \lambda$.

Lemma 3.1 Let $\lambda>0$ and $x^{0} \in \mathbb{R}^{n}$. There is a nonvanishing real function $u \in$ $C^{\infty}\left(\overline{B\left(x^{0}, r\right)}\right)$ solving $\left(\Delta+\lambda^{2}\right) u=0$ in $B\left(x^{0}, r\right)$ if and only if $r<c_{n} / \lambda$, where $c_{n}$ denotes the first positive zero of the Bessel function $J_{\frac{n-2}{2}}$.

Proof By translation invariance, we may assume $x^{0}=0$, and replacing $u(x)$ by $u(\lambda x)$, we may assume $\lambda=1$. We consider radial solutions $v=v(r)$ of $(\Delta+1) v=0$ in $\mathbb{R}^{n}$. Writing the Laplacian in polar coordinates, we see that $v$ should satisfy

$$
v^{\prime \prime}(r)+\frac{n-1}{r} v^{\prime}(r)+v(r)=0 \quad \text { in } \mathbb{R}_{+} .
$$

The substitution $v(r)=r^{\frac{2-n}{2}} w(r)$ leads to the Bessel equation

$$
r^{2} w^{\prime \prime}(r)+r w^{\prime}(r)+\left(r^{2}-\left(\frac{n-2}{2}\right)^{2}\right) w(r)=0 \quad \text { in } \mathbb{R}_{+} .
$$

Since $v(r)$ should be bounded near $r=0$, one must have $v(r)=r^{\frac{2-n}{2}} J_{\frac{n-2}{2}}(r)$ (up to a scalar multiple). Thus, there is a positive solution in $B\left(0, c_{n}\right)$.

For the converse, we argue by contradiction and suppose that $w \in C^{\infty}\left(\overline{B_{r}}\right)$ is positive and solves $(\Delta+1) w=0$ in $B_{r}$ for some $r \geq c_{n}$. We now use the fact that if a Schrödinger equation has a nonvanishing solution, then it can be reduced to a divergence form equation with no zero-order term. Writing $\gamma=w^{2}$, we see that

$$
(\Delta+1) u=0 \quad \text { in } B_{r} \quad \Longleftrightarrow \quad \operatorname{div}\left(\gamma \nabla\left(\gamma^{-1 / 2} u\right)\right)=0 \quad \text { in } B_{r} .
$$

Now since $r \geq c_{n}$, taking $u$ to be the radial solution $v$ above shows that $\gamma^{-1 / 2} u$ is positive in $B\left(0, c_{n}\right)$ but becomes zero on $\partial B\left(0, c_{n}\right)$. By the maximum principle for the equation $\operatorname{div}(\gamma \nabla \cdot)=0$, the maximum of $\gamma^{-1 / 2} u$ in $\overline{B\left(0, c_{n}\right)}$ should be attained at the boundary. This is a contradiction. 
We now turn to the question of determining if there is a real-valued solution of ( $\Delta+$ $\left.\lambda^{2}\right) u_{0}=0$ in $\mathbb{R}^{n}$ that is nonvanishing on $\partial D$. The following remark shows that this may be false when $\lambda>0$ is an eigenvalue of $-\Delta$ in $D$.

Remark 3.2 Let $D=B\left(0, c_{n}\right)$ where $c_{n}$ is as in Lemma 3.1, and suppose $u_{0}$ is real and solves $\left(\Delta+\lambda^{2}\right) u_{0}=0$ in $\mathbb{R}^{n}$. Let $v \in H_{0}^{1}(D)$ be the radial solution in the proof of Lemma 3.1 with $v>0$ in $D$. Since $\left.v\right|_{\partial D}=0$, one has

$$
\int_{\partial D} u_{0} \partial_{\nu} v d S=\int_{D}\left(u_{0} \Delta v-\left(\Delta u_{0}\right) v\right) d x=0 .
$$

However, $\partial_{\nu} v<0$ on $\partial D$, which implies that $u_{0}$ must change sign on $\partial D$. The same argument works for any sufficiently regular $D$ if $\lambda$ is the first Dirichlet eigenvalue and $\partial D$ is connected.

The next result shows that if $\lambda$ is not a Dirichlet eigenvalue in $D$, there is a solution $u_{0}$ which is positive on $\partial D$.

Proposition 3.3 Let $D \subset \mathbb{R}^{n}$ be a bounded Lipschitz domain if $n=2,3$ (resp. $C^{1}$ domain if $n \geq 4$ ) so that $\mathbb{R}^{n} \backslash \bar{D}$ is connected. Suppose that $\lambda>0$ is not a Dirichlet eigenvalue of $-\Delta$ in $D$. Then, there is a real-valued $u_{0}$ solving $\left(\Delta+\lambda^{2}\right) u_{0}=0$ in $\mathbb{R}^{n}$ so that $u_{0}$ is positive on $\partial D$.

We will prove the above result in two steps. First, we show that there is $v \in W^{1, p}(D)$ for some $p>n$ such that $\left(\Delta+\lambda^{2}\right) v=0$ in $D$ and $\left.v\right|_{\partial D}$ is positive. Then, we apply a Runge approximation property, showing that $v$ can be approximated by functions $\left.u\right|_{D}$ where $u$ solves $\left(\Delta+\lambda^{2}\right) u=0$ in $\mathbb{R}^{n}$. This kind of property is classical for second-order elliptic equations, and it follows from the unique continuation property. See $[34,39]$ for the original results in bounded domains and [45] for further references.

Approximation by Helmholtz solutions in $\mathbb{R}^{n}$ has been used in scattering theory at least with respect to $L^{2}$ norms, see e.g., [54] and references therein. We need a corresponding approximation result in the $C(\bar{D})$ norm. This is more involved than approximation in $L^{2}$ (for analytic functions this would correspond to Mergelyan's theorem instead of Runge's theorem). In order to achieve this, we will assume some regularity on $D$ and work with Sobolev norms instead.

Below, we say that $D$ is a $C^{0}$ domain if it is locally the region above the graph of a continuous function, and we define $H^{1, p}(D)=\left\{\left.u\right|_{D}: u \in W^{1, p}\left(\mathbb{R}^{n}\right)\right\}$ with the quotient norm $\|v\|_{H^{1, p}(D)}=\inf \left\{\|u\|_{W^{1, p}\left(\mathbb{R}^{n}\right)}:\left.u\right|_{D}=v\right\}$. The space $H^{1, p}(D)$, defined via restriction, coincides with the standard Sobolev space $W^{1, p}(D)$ whenever $D$ is a $W^{1, p}$ extension domain. The following version of the Runge approximation property will be relevant for us. In the rest of this section, we allow functions to be complex valued.

Proposition 3.4 Let $1<p<\infty$, let $\lambda>0$, and let $D \subset \mathbb{R}^{n}$ be a bounded $C^{0}$ domain such that $\mathbb{R}^{n} \backslash \bar{D}$ is connected. Given any $v \in H^{1, p}(D)$ (possibly complex valued) with $\left(\Delta+\lambda^{2}\right) v=0$ in $D$, there exist $u_{j}$ solving $\left(\Delta+\lambda^{2}\right) u_{j}=0$ in $\mathbb{R}^{n}$ so that

$$
\left\|u_{j}-v\right\|_{H^{1, p}(D)} \rightarrow 0 \quad \text { as } j \rightarrow \infty .
$$

If $v$ is real valued, then so are $u_{j}$.

The proof of Proposition 3.3 follows rather easily: 
Proof of Proposition 3.3 Since $\lambda$ is not a Dirichlet eigenvalue in $D$, there is a real-valued solution $v=1+w$ of $\left(\Delta+\lambda^{2}\right) v=0$ in $D$ where $w \in H_{0}^{1}(D)$ is the unique solution of

$$
\left(\Delta+\lambda^{2}\right) w=-\lambda^{2} \quad \text { in } D .
$$

We claim that $v \in W^{1, p}(D)$ for some $p>n$. Note that the $W^{1, p}(D)$ and $H^{1, p}(D)$ norms are equivalent since Lipschitz domains are $W^{1, p}$ extension domains. Now, if $v \in W^{1, p}(D)$ for some $p>n$, then by Proposition 3.4, there are global solutions $u_{j}$ such that

$$
\left\|u_{j}-1\right\|_{C(\partial D)} \leq\left\|u_{j}-v\right\|_{C(\bar{D})} \leq C\left\|u_{j}-v\right\|_{H^{1, p}(D)} \rightarrow 0
$$

where we used the Sobolev embedding. This shows the existence of a global solution that is positive on $\partial D$.

To prove that $v \in W^{1, p}(D)$ for some $p>n$, we note that $w \in H_{0}^{1}(D)$ solves

$$
\Delta w=-\lambda^{2} v \quad \text { in } D .
$$

By Sobolev embedding, the right-hand side is in $L^{\frac{2 n}{n-2}}(D)$ for $n \geq 3$, and in $L^{r}(D)$ for any $r<\infty$ for $n=2$. In particular, the right-hand side is in $W^{-1, r}(D)$ for any $r<\infty$ for $n=2,3,4$ and for $r=\frac{2 n}{n-4}$ for $n \geq 5$. Since $D$ is Lipschitz, by [29, Theorem 1.1], one has $w \in W^{1, p}(D)$ for some $p>3$ if $n=2$, 3. This proves the claim for Lipschitz domains in dimensions $n=2$, 3. For $C^{1}$ domains in dimensions $n \geq 4$, using [29, Theorem 1.1], which holds with $p_{0}=1$ in $C^{1}$ domains, shows that $w \in W^{1, \frac{2 n}{n-4}}(D)$. Returning to (3.1), noting that the right-hand side has more regularity, and iterating this argument shows that $v \in W^{1, p}(D)$ for all $p<\infty$ in the case of $C^{1}$ domains.

To prove Proposition 3.4, it is convenient to introduce the operator

$$
P(\lambda): C^{\infty}\left(S^{n-1}\right) \rightarrow C^{\infty}\left(\mathbb{R}^{n}\right), \quad P(\lambda) f(x)=\int_{S^{n-1}} e^{i \lambda x \cdot \omega} f(\omega) d \omega .
$$

Functions $u=P(\lambda) f$ are called Herglotz waves, and they are particular solutions of $\left(\Delta+\lambda^{2}\right) u=0$ in $\mathbb{R}^{n}$. One can think of $f$ as a certain boundary value at infinity for $u$, and of $P(\lambda)$ as a Poisson integral that gives the solution of $\left(\Delta+\lambda^{2}\right) u=0$ having boundary value $f$ at infinity.

The following proof, modeled after [42,52], will show that restrictions of Herglotz waves to $D$ are dense in the set of all Helmholtz solutions in $H^{1, p}(D)$.

Proof of Proposition 3.4 By the Hahn-Banach theorem, it is enough to prove that any bounded linear functional $\ell$ on $H^{1, p}(D)$ that vanishes on $\left\{\left.P(\lambda) f\right|_{D} ; f \in C^{\infty}\left(S^{n-1}\right)\right\}$ must also vanish on $\left\{v \in H^{1, p}(D) ;\left(\Delta+\lambda^{2}\right) v=0\right.$ in $\left.D\right\}$. We define a functional

$$
\ell_{1}: W^{1, p}\left(\mathbb{R}^{n}\right) \rightarrow \mathbb{C}, \quad \ell_{1}(u)=\ell\left(\left.u\right|_{D}\right) .
$$

Then, $\ell_{1}$ is bounded on $W^{1, p}\left(\mathbb{R}^{n}\right)$, and by duality

$$
\ell_{1}(u)=(u, \mu)
$$

for some $\mu \in W^{-1, p^{\prime}}\left(\mathbb{R}^{n}\right)$, where $\frac{1}{p}+\frac{1}{p^{\prime}}=1$ and $(\cdot, \cdot)$ is the sesquilinear distributional pairing in $\mathbb{R}^{n}$. Clearly $\mu=0$ in $\mathbb{R}^{n} \backslash \bar{D}$, so $\mu$ is a compactly supported distribution. Thus, the condition $\ell\left(\left.P(\lambda) f\right|_{D}\right)=0$ for all $f \in C^{\infty}\left(S^{n-1}\right)$ implies that

$$
(P(\lambda) f, \mu)=0 \quad \text { for all } f \in C^{\infty}\left(S^{n-1}\right) .
$$


Let $G_{\lambda}$ be the outgoing fundamental solution of $\Delta+\lambda^{2}$, given by

$$
G_{\lambda}(x)=c_{n, \lambda}|x|^{-\frac{n-2}{2}} H_{\frac{n-2}{2}}^{(1)}(\lambda|x|)
$$

where $H_{v}^{(1)}$ is the Hankel function (see [55, Section 1.2.3]), and let $w$ be the distribution

$$
w=G_{\lambda} * \mu .
$$

Then, $w$ is a distributional solution of

$$
\left(\Delta+\lambda^{2}\right) w=\mu \text { in } \mathbb{R}^{n} .
$$

By elliptic regularity $w \in W_{\text {loc }}^{1, p^{\prime}}\left(\mathbb{R}^{n}\right)$ and $w$ is smooth outside $\bar{D}$, with the expression

$$
w(x)=\left(G_{\lambda}(x-\cdot), \mu\right), \quad x \in \mathbb{R}^{n} \backslash \bar{D} .
$$

Let $f \in C^{\infty}\left(S^{n-1}\right)$ and $u=P(\lambda) f \in C^{\infty}\left(\mathbb{R}^{n}\right)$. By (3.2) and the fact that $\mu$ has compact support, we have

$$
0=(u, \mu)=\lim _{r \rightarrow \infty}(u, \mu)_{B_{r}}
$$

where $(\cdot, \cdot)_{B_{r}}$ is the sesquilinear distributional pairing in $B_{r}$. We wish to use that $\mu=$ $\left(\Delta+\lambda^{2}\right) w$. Since $w$ is not smooth in $\bar{D}$, we introduce a cutoff function $\chi \in C_{c}^{\infty}\left(\mathbb{R}^{n}\right)$ with $0 \leq \chi \leq 1$ and $\chi=1$ near $\bar{D}$. Writing $u=\chi u+(1-\chi) u$ and using that everything is smooth outside $\bar{D}$, we obtain from (3.4) that

$$
\begin{aligned}
0= & \lim _{r \rightarrow \infty}\left(\left(\chi u,\left(\Delta+\lambda^{2}\right) w\right)_{B_{r}}+\left((1-\chi) u,\left(\Delta+\lambda^{2}\right) w\right)_{B_{r}}\right] \\
= & \lim _{r \rightarrow \infty}\left[\left(\left(\Delta+\lambda^{2}\right)(\chi u), w\right)_{B_{r}}+\left(\left(\Delta+\lambda^{2}\right)((1-\chi) u), w\right)_{B_{r}}\right. \\
& \left.+\int_{\partial B_{r}}\left(u \overline{\partial_{\nu} w}-\left(\partial_{\nu} u\right) \bar{w}\right) d S\right] .
\end{aligned}
$$

Since $\left(\Delta+\lambda^{2}\right) u=0$, this reduces to

$$
\lim _{r \rightarrow \infty} \int_{\partial B_{r}}\left(u \overline{\partial_{\nu} w}-\left(\partial_{\nu} u\right) \bar{w}\right) d S=0 .
$$

Writing $x=r \theta$ where $r \geq 0$ and $\theta \in S^{n-1}$, the function $u=P(\lambda) f$ has the asymptotics

$$
\begin{gathered}
u(r \theta)=c_{n, \lambda}^{\prime} r^{-\frac{n-1}{2}}\left[e^{i \lambda r} f(\theta)+i^{n-1} e^{-i \lambda r} f(-\theta)\right]+O\left(r^{-\frac{n+1}{2}}\right), \\
\partial_{r} u(r \theta)=c_{n, \lambda}^{\prime} r^{-\frac{n-1}{2}} i \lambda\left[e^{i \lambda r} f(\theta)-i^{n-1} e^{-i \lambda r} f(-\theta)\right]+O\left(r^{-\frac{n+1}{2}}\right),
\end{gathered}
$$

as $r \rightarrow \infty$ (see [41, Section 1.3]). We wish to study similar asymptotics for the outgoing function $w$. Using (3.3) and asymptotics for the Hankel function, we see that (as in [55, Section 1.2.3])

$$
\begin{aligned}
w(r \theta) & =c_{n, \lambda}^{\prime \prime} r^{-\frac{n-1}{2}} e^{i \lambda r} \hat{\mu}(\lambda \theta)+O\left(r^{-\frac{n+1}{2}}\right), \\
\partial_{r} w(r \theta) & =c_{n, \lambda}^{\prime \prime} r^{-\frac{n-1}{2}} i \lambda e^{i \lambda r} \hat{\mu}(\lambda \theta)+O\left(r^{-\frac{n+1}{2}}\right)
\end{aligned}
$$

where $\hat{\mu} \in C^{\infty}\left(\mathbb{R}^{n}\right)$ is the Fourier transform of the compactly supported distribution $\mu$. Above $c_{n, \lambda}, c_{n, \lambda}^{\prime}$ and $c_{n, \lambda}^{\prime \prime}$ are nonzero constants. 
Inserting the asymptotics for $u$ and $w$ into (3.5) and noting that the terms containing $f(-\theta)$ cancel yields that

$$
\int_{S^{n-1}} f(\theta) \overline{\hat{\mu}(\lambda \theta)} d \theta=0 .
$$

Since this is true for all $f \in C^{\infty}\left(S^{n-1}\right)$, we must have $\hat{\mu}(\lambda \theta)=0$. In particular, $w$ has the asymptotics

$$
w(r \theta)=O\left(r^{-\frac{n+1}{2}}\right), \quad \partial_{r} w(r \theta)=O\left(r^{-\frac{n+1}{2}}\right) .
$$

Since $w$ is outgoing and satisfies $\left(\Delta+\lambda^{2}\right) w=0$ in $\mathbb{R}^{n} \backslash \bar{D}$, the Rellich uniqueness theorem (see e.g., [28]) implies that $w=0$ outside a large ball. Since $\mathbb{R}^{n} \backslash \bar{D}$ is connected, the unique continuation principle gives that $w=0$ in $\mathbb{R}^{n} \backslash \bar{D}$.

We have now proved that the condition (3.2) implies that

$$
\mu=\left(\Delta+\lambda^{2}\right) w \quad \text { in } \mathbb{R}^{n}
$$

for some $w \in W^{1, p^{\prime}}\left(\mathbb{R}^{n}\right)$ vanishing in $\mathbb{R}^{n} \backslash \bar{D}$. Now let $v \in H^{1, p}(D)$ be any solution of $\left(\Delta+\lambda^{2}\right) v=0$ in $D$, and let $\tilde{v} \in W^{1, p}\left(\mathbb{R}^{n}\right)$ be an extension of $v$. Then, we have

$$
\ell(v)=\ell_{1}\left(\left.\tilde{v}\right|_{D}\right)=(\tilde{v}, \mu)=\left(\tilde{v},\left(\Delta+\lambda^{2}\right) w\right)=\left(\left(\Delta+\lambda^{2}\right) \tilde{v}, w\right) .
$$

Since $D$ is a bounded $C^{0}$ domain and since $w \in W^{1, p^{\prime}}\left(\mathbb{R}^{n}\right)$ vanishes in $\mathbb{R}^{n} \backslash \bar{D}$, there are $w_{j} \in C_{c}^{\infty}(D)$ with $w_{j} \rightarrow w$ in $W^{1, p^{\prime}}\left(\mathbb{R}^{n}\right)$ (this is proved as in [40, Theorem 3.29]). It follows that

$$
\ell(v)=\lim _{j \rightarrow \infty}\left(\left(\Delta+\lambda^{2}\right) \tilde{v}, w_{j}\right)=0
$$

since $\left(\Delta+\lambda^{2}\right) \tilde{v}=0$ in $D$. This concludes the proof.

\section{Free boundary methods}

By arguments from Sect. 1.3, we know that $u=u_{q}-u_{0}$ satisfies the equation (see (1.11)$(1.12))$

$$
\Delta u=f(x) \chi_{\{u \neq 0\}} \quad \text { in } B\left(x^{0}, r\right),
$$

where we may assume that $f(x)>0$ in some neigborhood of $x^{0} \in \partial\{u \neq 0\}$. The above equation has been treated extensively in the literature, and all regularity aspects of the problem are resolved and sorted out; see e.g., [4] and the references therein. Theorem 1.5 follows directly from [4, Theorem 1.3], and the proof of Theorem 1.6 is sketched below. Theorem 1.4 in the case where $h$ is Dini or Lipschitz continuous follows from these results, and the higher regularity results follow from the method of [32] (see also [44, Section 6.4]).

We shall now give classical examples of singularities that can appear in the obstacle problem.

Example ([32,47-49]) We recall from [32, page 387-390] an explicit example of cusps appearing in the free boundary. These cusps are represented by the curves

$$
x_{2}= \pm x_{1}^{\mu / 2}, \quad 0 \leq x_{1} \leq 1,
$$

where $\mu=4 k+1,(k=1,2, \cdots)$ gives nonnegative solutions and $\mu=4 k+3,(k=0,1, \cdots)$ gives solutions that become negative on the negative $x_{1}$-axis and near the origin. The solution is defined locally by

$$
u(x)=x_{2}^{2}-\frac{2}{1+\mu / 2} \rho^{1+\mu / 2} \sin (1+\mu / 2) \theta+\cdots, \quad x \in \Omega,|x|<\epsilon,
$$


for $\epsilon$ small. Here, we have used both real and complex notation

$$
x=\left(x_{1}, x_{2}\right), \quad z=\rho e^{i \theta}, \quad 0 \leq \theta \leq 2 \pi .
$$

Also the domain $\Omega$ is the image of the set

$$
\{z:|z|<1, \operatorname{Im} z>0\}
$$

under the conformal mapping $f(z)=z^{2}+i z^{\mu}$.

Proof of Theorem 1.6 The proof of Theorem 1.6 when $h \in C^{0,1}\left(B\left(x^{0}, r\right)\right)$ (i.e., case (1)) is somehow hidden in [15] (see their proof of Main Theorem), where it is proven that the singular set of the free boundary lies in a $C^{1}$-manifold. In particular, this means that whenever we blow up a solution at a singular free boundary point through any sequence $u\left(r_{j} x+x^{0}\right) / r_{j}^{2}$ (here $u=u_{q}-u_{0}$ satisfies (4.1)), it will converge to a fixed polynomial $p(x)$, with the free boundary $\{p=\nabla p=0\}$, and regardless of the sequence $\left\{r_{j}\right\}$. This in particular implies that the limiting free boundary lies in a plane (or lower dimensional plane) which after translation and rotation we assume it is $\left\{x_{n}=0\right\}$. From here, it follows that the free boundary approaches this plane tangentially, whence the statement $b$ ) follows whenever the free boundary has a cusp at $x^{0}$.

In case $x^{0}$ is not a cusp point, then by Theorem 1.5 in a vicinity of $x^{0}$ the free boundary is $C^{1}$, and $u \geq 0$, and obviously $\partial_{e} u \geq 0$ in a smaller neighbourhood of $x^{0}$. By results of [3] (see section 1.4.2), the free boundary is $C^{1, \alpha}$, and Theorem 1.6 is proved in case (1).

To prove Theorem 1.6 in the case (2), we work with $v=\partial_{e} u$, where by the assumption $e=\nabla u_{0}\left(x^{0}\right) \neq \mathbf{0}$. Since $u_{q}=u_{0}$ in $D^{c}$, we also have $\nabla u_{q}\left(x^{0}\right) \neq \mathbf{0}$. As before with $u=u_{q}-u_{0}$, we have $-\left(\Delta+\lambda^{2}\right) \partial_{e} u=\partial_{e}\left(h u_{q}\right) \chi_{D}=\left(\partial_{e} h u_{q}+h \partial_{e} u_{q}\right) \chi_{D}$ close to $x^{0}$. Since $u_{0}=u_{q}=0$ on $\partial D \cap B_{r}\left(x^{0}\right)$ and $\partial_{e} u_{q}\left(x^{0}\right)>0$ and $h\left(x^{0}\right) \neq 0$, we have that $\partial_{e} u$ satisfies the hypothesis of Theorem 1.6 case (1), and hence the result follows.

\section{Acknowledgements}

M. S. was supported by the Academy of Finland (Finnish Centre of Excellence in Inverse Modelling and Imaging, Grant Numbers 312121 and 309963) and by the European Research Council under Horizon 2020 (ERC CoG 770924). H. Sh. was supported in part by Swedish Research Council.

Funding

Open access funding provided by University of Jyväskylä (JYU).

\section{Author details}

${ }^{1}$ Department of Mathematics and Statistics, University of Jyväskylä, Jyväskylä, Finland, ${ }^{2}$ Department of Mathematics, KTH Royal Institute of Technology, Stockholm, Sweden.

Received: 29 June 2021 Accepted: 25 August 2021 Published online: 5 October 2021

\section{References}

1. Alessandrini, G.: Generic uniqueness and size estimates in the inverse conductivity problem with one measurement, Matematiche (Catania) 54 (1999), no. suppl., 5-14. Boundary value problems for elliptic and parabolic operators (Catania, 1998)

2. Alessandrini, G., Isakov, V.: Analyticity and uniqueness for the inverse conductivity problem. Rend. Istit. Mat. Univ. Trieste 28 (1996), no. 1-2, 351-369 (1997) (English, with English and Italian summaries)

3. Allen, M., Shahgholian, H.: A new boundary Harnack principle (equations with right hand side). Arch. Ration. Mech. Anal. 234(3), 1413-1444 (2019). https://doi.org/10.1007/s00205-019-01415-3

4. Andersson, J., Lindgren, E., Shahgholian, H.: Optimal regularity for the no-sign obstacle problem. Commun. Pure Appl. Math. 66(2), 245-262 (2013). https://doi.org/10.1002/cpa.21434

5. Arridge, S.R., Schotland, J.C.: Optical tomography: forward and inverse problems. Inverse Probl. 2009). https://doi.org/ 10.1088/0266-5611/25/12/123010

6. Athanasopoulos, I., Caffarelli, L.A., Salsa, S.: The free boundary in an inverse conductivity problem. J. Reine Angew. Math. 534, 1-31 (2001). https://doi.org/10.1515/crll.2001.033 
7. Blåsten, E.: Nonradiating sources and transmission eigenfunctions vanish at corners and edges. SIAM J. Math. Anal. 50(6), 6255-6270 (2018). https://doi.org/10.1137/18M1182048

8. Blåsten, E., Liu, H.: On vanishing near corners of transmission eigenfunctions. J. Funct. Anal. 273(11), 3616-3632 (2017). https://doi.org/10.1016/j.jfa.2017.08.023

9. Blåsten, E., Liu, H.: Scattering by curvatures, radiationless sources, transmission eigenfunctions and inverse scattering problems (2018), available at 1808.01425

10. Blåsten, E., Liu, H.: Recovering piecewise constant refractive indices by a single far-field pattern. Inverse Problems 36(8), 085005 (2020). https://doi.org/10.1088/1361-6420/ab958f

11. Blåsten, E., Päivärinta, L., Sylvester, J.: Corners always scatter. Commun. Math. Phys. 331(2), 725-753 (2014). https:// doi.org/10.1007/s00220-014-2030-0

12. Blåsten, E., Vesalainen, E.V.: Non-scattering energies and transmission eigenvalues in Hn. Ann. Acad. Sci. Fenn. Math. 45(1), 547-576 (2020). https://doi.org/10.5186/aasfm.2020.4522

13. Caffarelli, L.A.: The regularity of free boundaries in higher dimensions. Acta Math. 139(3-4), 155-184 (1977). https:// doi.org/10.1007/BF02392236

14. Caffarelli, L.A., Friedman, A.: The free boundary in the Thomas-Fermi atomic model. J. Differ. Equ. 32(3), 335-356 (1979). https://doi.org/10.1016/0022-0396(79)90038-X. (References [14, 20, 33,50] are given in list but not cited in text. Please cite in text or delete from list.)

15. Caffarelli, L.A., Shahgholian, H.: The structure of the singular set of a free boundary in potential theory. Izv. Nats. Akad. Nauk Armenii Mat. 39(2), 43-58 (2004)

16. Cakoni, F., Colton, D., Haddar, H.: Inverse scattering theory and transmission eigenvalues, CBMS-NSF Regional Conference Series in Applied Mathematics, vol. 88. Society for Industrial and Applied Mathematics (SIAM), Philadelphia, PA (2016)

17. Cakoni, F., Haddar, H.: Transmission eigenvalues in inverse scattering theory, Inverse problems and applications: inside out. II, Math. Sci. Res. Inst. Publ., vol. 60, Cambridge Univ. Press, Cambridge, 2013, pp. 529-580

18. Cakoni, F., Vogelius, M.S.: Singularities almost always scatter: regularity results for non-scattering inhomogeneities (2021)

19. Colton, D., Kress, R.: Inverse acoustic and electromagnetic scattering theory. Applied Mathematical Sciences, vol. 93, Springer, Cham, 2019. Fourth edition

20. Colton, D., Päivärinta, L., Sylvester, J.: The interior transmission problem. Inverse Probl. Imaging 1(1), 13-28 (2007). https://doi.org/10.3934/ipi.2007.1.13

21. Davis, P.J.: The Schwarz function and its applications. The Mathematical Association of America, Buffalo, N. Y., 1974. The Carus Mathematical Monographs, No. 17

22. Elschner, J., Hu, G.: Corners and edges always scatter. Inverse Problems 31(1), 015003 (2015). https://doi.org/10.1088/ 0266-5611/31/1/015003

23. Elschner, J., Hu, G.: Acoustic scattering from corners, edges and circular cones. Arch. Ration. Mech. Anal. 228(2), 653-690 (2018). https://doi.org/10.1007/s00205-017-1202-4

24. Figalli, A., Kim, S., Shahgholian, H. (2020), available at 2012.15499

25. Figalli, A., Shahgholian, H.: An overview of unconstrained free boundary problems. Philos. Trans. Roy. Soc. A $\mathbf{3 7 3}$ (2050), 11 (2015). https://doi.org/10.1098/rsta.2014.0281

26. Gell-Redman, J., Hassell, A.: Potential scattering and the continuity of phase-shifts. Math. Res. Lett. 19(3), 719-729 (2012). https://doi.org/10.4310/MRL.2012.v19.n3.a15

27. Hu, G., Salo, M., Vesalainen, E.V.: Shape identification in inverse medium scattering problems with a single far-field pattern. SIAM J. Math. Anal. 48(1), 152-165 (2016). https://doi.org/10.1137/15M1032958

28. Hörmander, L.: Lower bounds at infinity for solutions of differential equations with constant coefficients. Israel J. Math. 16, 103-116 (1973). https://doi.org/10.1007/BF02761975

29. Jerison, D., Kenig, C.E.: The inhomogeneous Dirichlet problem in Lipschitz domains. J. Funct. Anal. 130(1), 161-219 (1995). https://doi.org/10.1006/jfan.1995.1067

30. Kim, S., Lee, K.-A., Shahgholian, H.: An elliptic free boundary arising from the jump of conductivity. Nonlinear Anal. 161, 1-29 (2017). https://doi.org/10.1016/j.na.2017.05.010

31. Kim, S., Lee, K.-A., Shahgholian, H.: Nodal sets for "broken" quasilinear PDEs. Indiana Univ. Math. J. 68(4), 1113-1148 (2019). https://doi.org/10.1512/iumj.2019.68.7711

32. Kinderlehrer, D., Nirenberg, L.: Regularity in free boundary problems. Ann. Scuola Norm. Sup. Pisa CI. Sci. 4(4), 373-391 (1977)

33. Kusiak, S., Sylvester, J.: The scattering support. Commun. Pure Appl. Math. 56(11), 1525-1548 (2003). https://doi.org/ 10.1002/cpa.3038

34. Lax, P.D.: A stability theorem for solutions of abstract differential equations, and its application to the study of the local behavior of solutions of elliptic equations. Commun. Pure Appl. Math. 9, 747-766 (1956). https://doi.org/10. 1002/cpa.3160090407

35. Li, L., Hu, G., Yang, J.: Interface with weakly singular points always scatter. Inverse Problems 34(7), 075002 (2018) https://doi.org/10.1088/1361-6420/aabe56

36. Liu, H.: On local and global structures of transmission eigenfunctions and beyond (2020), available at 2008.03120

37. Liu, H., Tsou, C.-H.: Stable determination of polygonal inclusions in Calderón's problem by a single partial boundary measurement. Inverse Problems 36(8), 085010 (2020). https://doi.org/10.1088/1361-6420/ab9d6b

38. Logunov, A., Malinnikova, E.: Review of Yau's conjecture on zero sets of Laplace eigenfunctions (2019), available at 1908.01639

39. Malgrange, B.: Existence et approximation des solutions des équations aux dérivées partielles et des équations de convolution. Ann. Inst. Fourier (Grenoble) 6 (1955/56), 271-355 (French)

40. McLean, W.: Strongly Elliptic Systems and Boundary Integral Equations. Cambridge University Press, Cambridge (2000)

41. Melrose, R.B.: Geometric Scattering Theory. Cambridge University Press, Cambridge (1995)

42. Päivärinta, L., Salo, M., Uhlmann, G.: Inverse scattering for the magnetic Schrödinger operator. J. Funct. Anal. 259(7), 1771-1798 (2010). https://doi.org/10.1016/j.fa.2010.06.002 
43. Päivärinta, L., Salo, M., Vesalainen, E.V.: Strictly convex corners scatter. Rev. Mat. Iberoam. 33(4), 1369-1396 (2017). https://doi.org/10.4171/RMl/975

44. Petrosyan, A., Shahgholian, H., Uraltseva, N.: Regularity of Free Boundaries in Obstacle-Type Problems, Graduate Studies in Mathematics, vol. 136. American Mathematical Society, Providence, RI (2012)

45. Rüland, A., Salo, M.: Quantitative Runge approximation and inverse problems. Int. Math. Res. Not. IMRN 20, 6216-6234 (2019). https://doi.org/10.1093/imrn/rnx301

46. Sakai, M.: Quadrature Domains. Lecture Notes in Mathematics, vol. 934. Springer, Berlin (1982)

47. Sakai, M.: Regularity of a boundary having a Schwarz function. Acta Math. 166(3-4), 263-297 (1991). https://doi.org/ 10.1007/BF02398888

48. Sakai, M.: Regularity of free boundaries in two dimensions. Ann. Scuola Norm. Sup. Pisa Cl. Sci. 20(3), 323-339 (1993)

49. Schaeffer, D.G.: Some examples of singularities in a free boundary. Ann. Scuola Norm. Sup. Pisa Cl. Sci. 4(1), 133-144 (1977)

50. Stein, E.M., Weiss, G.: Introduction to Fourier analysis on Euclidean spaces. Princeton University Press, Princeton, N.J., 1971. Princeton Mathematical Series, No. 32

51. Uhlmann, G.: 30 years of Calderón's problem, Séminaire Laurent Schwartz-Équations aux dérivées partielles et applications. Année 2012-2013, Sémin. Équ. Dériv. Partielles, École Polytech., Palaiseau, 2014, pp. Exp. No. XIII, 25

52. Uhlmann, G., Vasy, A.: Fixed energy inverse problem for exponentially decreasing potentials. Methods Appl. Anal. 9(2), 239-247 (2002). https://doi.org/10.4310/MAA.2002.v9.n2.a2

53. Vogelius, M., Xiao, J.: Finiteness results concerning non-scattering wave numbers for incident plane- and Herglotz waves. SIAM J. Math. Anal. (2021), to appear

54. Weder, R.: Completeness of averaged scattering solutions and inverse scattering at a fixed energy. Commun. Partial Differ. Equ. 32(4-6), 675-691 (2007). https://doi.org/10.1080/03605300500530370

55. Yafaev, D.R.: Mathematical scattering theory, Mathematical Surveys and Monographs, vol. 158, American Mathematical Society, Providence, Rl, 2010. Analytic theory

56. Yeressian, K.: Obstacle problem with a degenerate force term. Anal. PDE 9(2), 397-437 (2016). https://doi.org/10. 2140/apde.2016.9.397

\section{Publisher's Note}

Springer Nature remains neutral with regard to jurisdictional claims in published maps and institutional affiliations. 